\title{
Spin transfer torque on magnetic insulators
}

\author{
Xingtao Jia ${ }^{1}$, Kai Liu ${ }^{1}$ and $\mathrm{Ke} \mathrm{XiA}^{1} \quad$ (a) And Gerrit E. W. BaueR ${ }^{2,3}$ \\ 1 Department of Physics, Beijing Normal University, Beijing 100875, China \\ 2 Institute for Materials Research, Tohoku University, Sendai 980-8577, Japan \\ Delft University of Technology, Kavli Institute of NanoScience, 2628 CJ Delft, The Netherlands
}

PACS $72.25 . \mathrm{Mk}$ - Spin transport through interfaces

PACS 72.10.- d - Theory of electronic transport; scattering mechanisms

PACS 85.75.-d - Magnetoelectronics; spintronics: devices exploiting spin polarized transport or integrated magnetic fields

\begin{abstract}
Recent experimental and theoretical studies focus on spin-mediated heat currents at interfaces between normal metals and magnetic insulators. We resolve conflicting estimates for the order of magnitude of the spin transfer torque by first-principles calculations. The spin mixing conductance $G^{\uparrow \downarrow}$ of the interface between silver and the insulating ferrimagnet Yttrium Iron Garnet (YIG) is dominated by its real part and of the order of $10^{14} \Omega^{-1} \mathrm{~m}^{-2}$, i.e. close to the value for intermetallic interface, which can be explained by a local spin model.
\end{abstract}

Introduction. - It has recently been reported that the magnetism of insulators can be actuated electrically and thermally by normal metal contacts [1,2, The material of choice is the ferrimagnet $\mathrm{Y}_{3} \mathrm{Fe}_{5} \mathrm{O}_{12}$ (YIG), because of its extremely small magnetic damping [3 5]. The lowlying excitations of magnetic insulators are spin waves, which carry heat and angular momentum [6]. Existing experiments use Pt contacts, which by means of the inverse spin Hall effect are effective spin current detectors [7]. Slonczewski [8] reports that the thermal spin transfer torque in magnetic nanopillars [9], can be much more efficient than the electrically generated spin torque in metallic structures.

The electrical and thermal injection of spin and heat currents into insulating magnets is governed by the spin transfer torque at the metal|insulator interface [11, 12], which is parameterized by the spin-mixing conductance $G^{\uparrow \downarrow}=e^{2} \operatorname{Tr}\left(\mathbf{I}-\mathbf{r}_{\uparrow}^{\dagger} \mathbf{r}_{\downarrow}\right) / h$, where $\mathbf{I}$ and $\mathbf{r}_{\sigma}$ are the unit matrix and the matrix of interface reflection coefficients for spin $\sigma$ spanned by the scattering channels at the Fermi energy of the metal 13. Crude approximations such as a Stoner model with spin-split conduction bands 11 and parameterized exchange between the itinerant metal electrons and local moments of the ferromagnet [1,8,12, have been used to estimate $G^{\uparrow \downarrow}$ for YIG interfaces 1 . Experi-

\footnotetext{
(a) E-mail: kexia@bnu.edu.cn

${ }^{1}$ The spin mixing conductance is governed by the reflection coefficients only and remains finite when the transmission coefficients van-
}

ments and initial theoretical estimates found very small spin torques that are at odds with Slonczewski's predictions [8].

Here we report calculations of the spin mixing conductance for the $\mathrm{Ag} \mid \mathrm{YIG}$ interface based on realistic electronic structures. Silver is a promising material 14 for non-local spin current detection [15, which should be more efficient than the inverse spin Hall effect in nanostructures. We demonstrate that the calculated $G^{\uparrow \downarrow}$ for the $\mathrm{Ag} \mid$ YIG interface is much larger than expected from the Stoner model and better described by local-moment exchange fields.

Free-electron model. - We start with a reference structure consisting of an $\mathrm{Ag}|\mathrm{FI}| \mathrm{Ag}(001)$ junction in which the ferromagnetic insulator (FI) is modeled by a spinsplit vacuum barrier, i.e., the free-electron Stoner model. The vacuum potential is chosen to be spin-split by 0.3 and $3.0 \mathrm{eV}$, whereas the barrier height is adjusted to 0.3 , $1.4,2.6$ and $2.85 \mathrm{eV}$, respectively. The barrier thickness $(1.2 \mathrm{~nm})$ is chosen here such that electron transmission is negligible. Table 1 lists the corresponding $G^{\uparrow \downarrow}$ of $\operatorname{Ag}|\mathrm{FI}| \mathrm{Ag}$. Both $\operatorname{Re} G^{\uparrow \downarrow}$ and $\operatorname{Im} G^{\uparrow \downarrow}$ decrease with increasing barrier height, as expected [11].

Band structure. - We calculate the electronic structure of YIG using the tight-binding linear-muffin-tin-

ish. This is not a breach of the scattering theory of transport,since the incoming and outgoing scattering states are well defined as propagating states in the metallic contacts. 
Table 1: Spin-dependent and spin mixing conductances of a $\mathrm{Ag}|\mathrm{FI}| \operatorname{Ag}(001)$ junction with different barrier heights and spin splitting $\Delta=0.3$ and $3.0 \mathrm{eV}$. The mixing conductances for the (111) orientation differs by less than $20 \%$. The Sharvin conductance $\left(\mathrm{G}^{S h}\right)$ of $\mathrm{Ag}(001)$ is $4.5 \times 10^{14} \Omega^{-1} \mathrm{~m}^{-2}$.

\begin{tabular}{|c|c|c|c|c|}
\hline$\overline{\overline{\text { Barrier }}}$ & $\overline{G^{\uparrow}} / G^{s h}$ & $\overline{G^{\downarrow} / G^{s h}}$ & $\overline{\operatorname{Re}} G^{\uparrow \downarrow} / G^{s h}$ & $\overline{\overline{\operatorname{Im}} G^{\text {} \downarrow} / G^{s}} h$ \\
\hline \multicolumn{5}{|c|}{$\Delta=0.3 \mathrm{eV}$} \\
\hline 0.3 & $6.3 \mathrm{E}-5$ & $5.1 \mathrm{E}-6$ & 0.009 & $-1.1 \mathrm{E}-1$ \\
\hline 1.4 & $3.3 \mathrm{E}-8$ & $7.1 \mathrm{E}-9$ & 0.003 & $-7.4 \mathrm{E}-2$ \\
\hline 2.6 & $3.5 \mathrm{E}-9$ & $1.3 \mathrm{E}-9$ & 0.001 & $-4.0 \mathrm{E}-2$ \\
\hline 2.85 & $0^{*}$ & 0 & 0.001 & $-5.1 \mathrm{E}-2$ \\
\hline \multicolumn{5}{|c|}{$\Delta=3.0 \mathrm{eV}$} \\
\hline 0.3 & 7.0E-6 & 0 & 0.15 & -0.45 \\
\hline 1.4 & $7.4 \mathrm{E}-10$ & 0 & 0.08 & -0.35 \\
\hline 2.6 & 0 & 0 & 0.05 & -0.28 \\
\hline 2.85 & 0 & 0 & 0.04 & -0.27 \\
\hline
\end{tabular}

orbital code in the augmented spherical wave approximation as implemented in the Stuttgart code [16 18 using the generalized gradient correction (GGA) to the local density approximation (LDA). The cubic lattice constant $a=12.2 \AA$ is chosen $1.6 \%$ smaller than the experimental one [19]. We use 136 additional empty spheres (ES) for better space filling and reduced overlap between neighboring atomic spheres. YIG is a ferrimagnetic insulator with band gap of $2.85 \mathrm{eV}$ 20, 21. Magnetism is carried by majority and minority spin Fe atoms (tetragonal $\mathrm{Fe}(\mathrm{T})$ and octahedral $\mathrm{Fe}(\mathrm{O})$ sites in Fig 1(a), respective.) with a net magnetic moment of $5 \mu_{B}$ per formula unit 19, 22, 24. The magnetic moments are 3.95 and $-4.06 \mu_{B}$ for majority and minority spin Fe atoms, respectively. Both $\mathrm{Y}$ and $\mathrm{O}$ atoms show small positive magnetic moments of 0.03 and $0.09 \mu_{B}$, respectively, while those on the empty spheres do not exceed $0.007 \mu_{B}$. The common problem of density-functional theory to predict the energy gap of insulators can be handled by an on-site Coulomb correction $(\mathrm{LDA} / \mathrm{GGA}+\mathrm{U})$ 25,26 or a scissor operator $(\mathrm{LDA} / \mathrm{GGA}+\mathrm{C})$ [27. Figure 1(b) is a plot of the band structure of GGA with a fundamental band gap of $0.33 \mathrm{eV}$ between the valence band edge of the majority-spin channel and conductance band edge of minority-spin channel. The GGA $+\mathrm{C}$ method can be used to increase the band gap depending on the scissor parameters C. A GGA+C band structure with a band gap of $\backsim 1.25 \mathrm{eV}$ is shown in Fig. 1(c). The GGA+U method applied to the YIG band structure using the parameters from Ref. [25,26] leads to the band structure plotted in 1(d) with the same energy gap $\backsim 1.25 \mathrm{eV}$.

While a visual comparison of the band structures in Figs. 11(c) and (d) assures the equivalence of the two methods, we can assess the differences quantitatively by comparing the effective masses at the band edges as shown in
Table 2: Band gap $\left(E_{g}\right)$ and effective masses (in unit of $m_{e}$ ) of the band structure of YIG at the $\Gamma$ point as calculated by the GGA, GGA+U, and GGA $+\mathrm{C}$ methods. $\mathrm{CB}$ and VB denote conductance and valence bands, respectively.

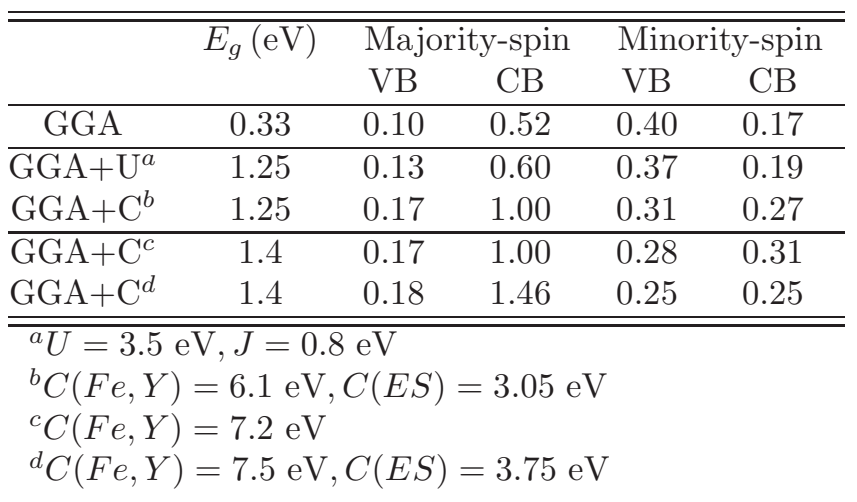

Table 2. For band gaps of $\sim 1.25 \mathrm{eV}$ the effective mass at the conductance band edge of majority-spin as obtained by the $\mathrm{GGA}+\mathrm{U}$ and $\mathrm{GGA}+\mathrm{C}$ methods differ by up to $67 \%$. This seems significant, but the effects on the mixing conductance, which is the quantity of our main interest here, is small, as discussed in the next section.

Ag $\mid$ YIG interface. - We study the spin mixing conductance in $\mathrm{Ag}|\mathrm{YIG}| \mathrm{Ag}$ with a $3 \times 3$ and $6 \times 6$ lateral supercell of fcc Ag to match a cubic YIG unit cell along the (001) and (111) directions with lattice mismatch $\sim 1 \%$. Interfaces can be classified according to their magnetic surface properties into three types of terminations. For the (001) texture, one cut is terminated by $\mathrm{Y}$ as well as majority and minority spin Fe atoms with compensated magnetic moment ("YFe-termination"). Another cut yields only majority Fe atoms at the interface ("Fe-termination") with total magnetic moment of $7.90 \mu_{B}$ per lateral unit cell. The third interface covered by $\mathrm{O}$ atoms is obtained by removing $\mathrm{Fe}$ and $\mathrm{Y}$ atoms from the YFe-termination. The oxygen layer is separated from adjacent Fe atoms by only $\sim 0.3 \AA$. Including the latter, the "O-termination" also corresponds to a net interface magnetic moment of $7.90 \mu_{B}$. The interfaces for the (111) direction can be classified analogously. The "YFe-termination" cut has now a net interface magnetic moment of $23.70 \mu_{B}$. The Fetermination contains now minority-spin $\mathrm{Fe}$ atoms with net magnetic moment of $-16.24 \mu_{B}$, while the O-terminated surface has the same magnetic moment when including the shallowly buried Fe layer.

We chose a YIG film of 4 unit cell layers, because its electric conductance does not exceed $10^{-10} e^{2} / h$ per unit cell. $G^{\uparrow \downarrow}$ is therefore governed solely by the single Ag|YIG interface.

First, we inspect $G^{\uparrow \downarrow}$ of $\mathrm{Ag} \mid \mathrm{YIG}$ interfaces computed with and without scissor corrections. We find that the difference of $\operatorname{Re} G^{\uparrow \downarrow}$ is as small as $21 \%$ when increasing the band gap of YIG from its GGA value of $0.33 \mathrm{eV}$ to 
(a)

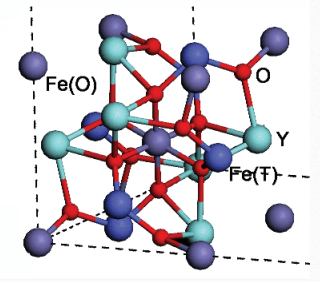

(c)

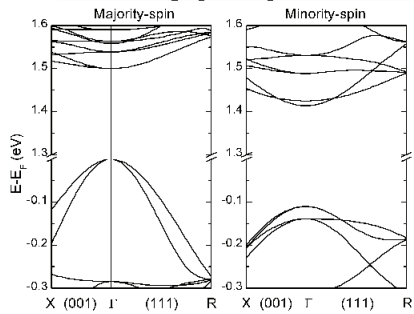

(b)

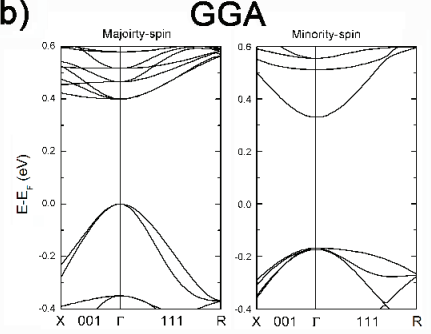

(d)

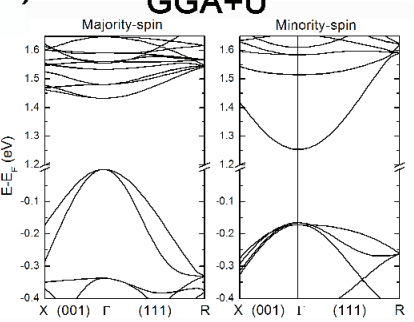

a)
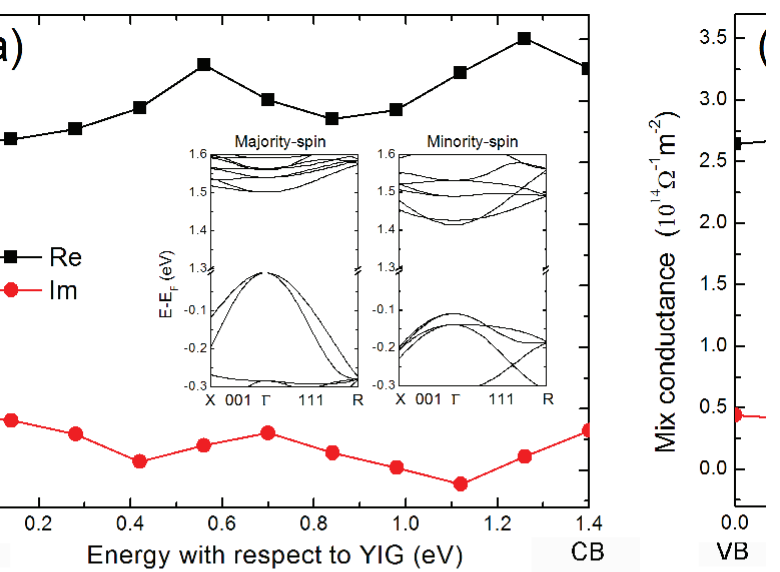

b)
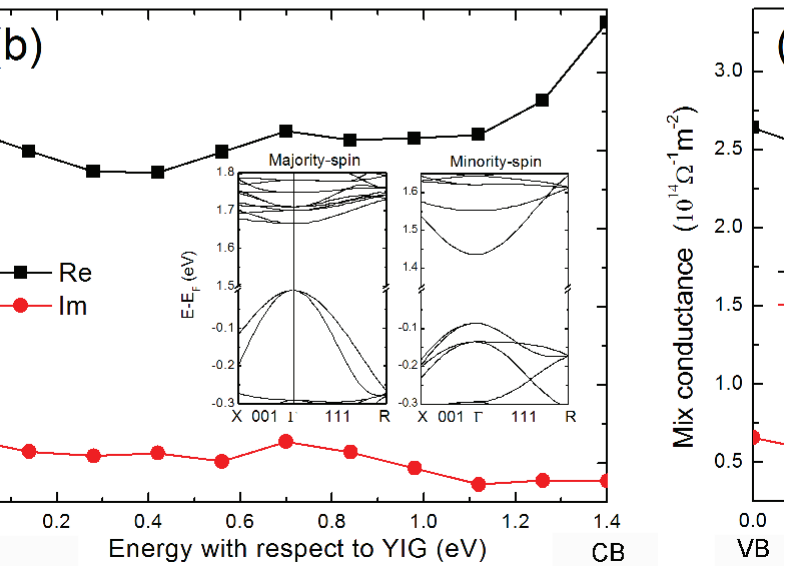

Fig. 1: (a): 1/8 of the cubic YIG cell; the full structure can be obtained by symmetry operations. Here, $\mathrm{Fe}(\mathrm{T})$ and $\mathrm{Fe}(\mathrm{O})$ are Fe atoms at tetragonal and octahedronal sites, respectively. (bd): Band structures of YIG with GGA, GGA+C, and GGA+U method with band gap of $0.33,1.25$, and $1.25 \mathrm{eV}$, respectively.

a GGA $+\mathrm{C}$ band gap of $2.1 \mathrm{eV}$ as shown in Table 3. We conclude that the precise band gap is a parameter that hardly affects the $G^{\uparrow \downarrow}$ of the Ag|YIG interface.

Table 3: Spin mixing conductance of Ag|YIG(001) with different YIG band gaps modulated by the GGA $+\mathrm{C}$ methods. We pin the Fermi level of $\mathrm{Ag}$ at mod-gap of YIG. $0.33 \mathrm{eV}$ is the band gap of GGA (without a scissor operator).

\begin{tabular}{lcccccc}
\hline \hline$E_{g}(\mathrm{eV})$ & 0.33 & 0.65 & 0.95 & 1.4 & 1.8 & 2.1 \\
\hline $\operatorname{Re} G^{\uparrow \downarrow}$ & & & & & & \\
$\left(10^{14} \Omega^{-1} \mathrm{~m}^{-2}\right)$ & 3.46 & 3.94 & 3.43 & 3.01 & 2.82 & 2.74 \\
\hline
\end{tabular}

By scanning the Fermi energy of Ag (or the YIG work function), we can obtain information similar to that when varying the band gap. Scanning the Ag Fermi energy from the valence to conductance band edges for a band gap of $1.4 \mathrm{eV}$, we obtain results equivalent to a mid-gap Fermi energy and band gaps varying from zero to $2.8 \mathrm{eV}$, but without changing the details of the band dispersion. In Figure 2 we plot the mixing conductance of Ag|YIG(001) with YFe termination as a function of YIG's work function. Here we consider two kinds of band dispersions with the same band gap of $1.4 \mathrm{eV}$ obtained by different scissor operator implementations as shown in Table 2 We find that the mixing conductance does not depend sensitively on (i) the YIG work function or interface potential barrier as well as (ii) the band dispersion when fixing the Fermi energy of $\mathrm{Ag}$ in the middle of the band gap of YIG; the difference in effective mass of $46 \%$ causes changes in

Fig. 2: Effect of band dispersion and band alignment on the spin mixing conductance of $\mathrm{Ag}|\mathrm{YIG}| \mathrm{Ag}(001)$ with $\mathrm{YFe}-$ termination. We use YIG with same band gap of $1.4 \mathrm{eV}$ with different implementations of scissor operator (a) $\mathrm{C}(\mathrm{Fe}, \mathrm{Y})=$ $7.2 \mathrm{eV}$; (b) $\mathrm{C}(\mathrm{Fe}, \mathrm{Y})=7.5 \mathrm{eV}$, and $\mathrm{C}(\mathrm{ES})=3.75 \mathrm{eV}$ to see the effect of band dispersion. We fix the Fermi energy of $\mathrm{Ag}$ while scanning the YIG work function.

$\operatorname{Re} G^{\uparrow \downarrow}$ of only $13 \%$. These deviations are within the error bars due to other approximations (see below). We therefore conclude that the transport properties in the present system are sufficiently well represented by the scissor operator or on-site Coulomb correction methods for the gap problem.

Besides the band alignment discussed in the previous paragraph, two more properties are difficult to compute self-consistently for large unit cells, viz. the atomic interface configuration and the ferromagnetic proximity effect: (i): We determine the distance between Ag|YIG by minimizing ASA overlap while keeping the space filled. We estimate that the differences in $G^{\uparrow \downarrow}$ for configurations with maximum and minimum ASA overlap is less than $30 \%$ (ii): We assess the ferromagnetic proximity effect by using the self-consistent electronic structure of $\mathrm{Ag}$ atom at the $\mathrm{Ag} \mid \mathrm{Fe}$ interface. We find that the $\mathrm{Ag}$ atoms closest to Fe acquire a magnetic moment of $0.025 \mu_{B}$ and the ef- 


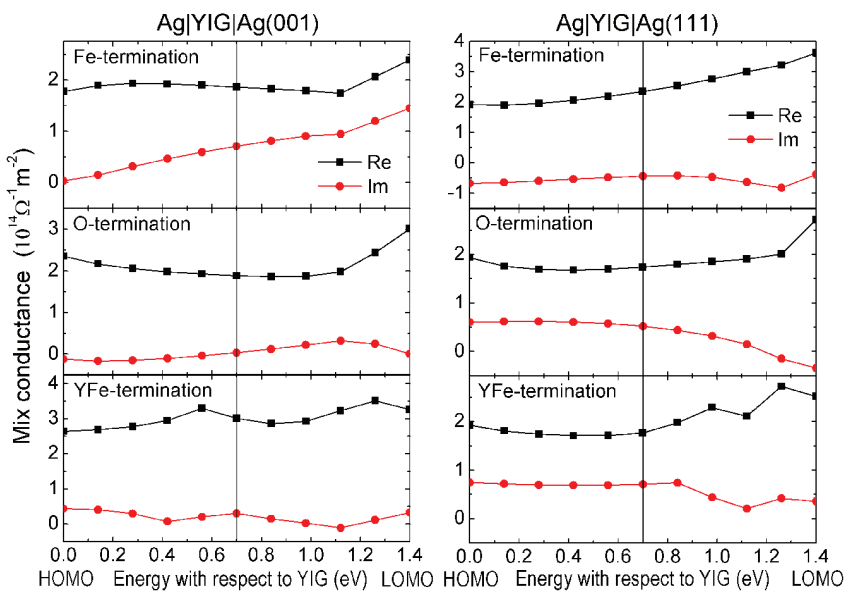

Fig. 3: Spin mixing conductance of $\mathrm{Ag} \mid \mathrm{YIG}(001)$ (left) and $\mathrm{Ag} \mid \mathrm{YIG}(111)$ (right) with a YIG band gap of $1.4 \mathrm{eV}$. We fix the Fermi energy of Ag while scanning the YIG work function.

fect is observable up to the 4 th Ag layer. The spin mixing conductance is found to be enhanced by about $10 \%$ in this system. In the following we disregard such an effect. From various checks of these and other issues, the magnitude of a possible systematic error in the mixing conductance is estimated to be $<40 \%$.

Results. - Fig. 3 summarizes our results for $G^{\uparrow \downarrow}$ of $\mathrm{Ag} \mid \mathrm{YIG}(111)$ and $\mathrm{Ag} \mid \mathrm{YIG}(001)$ junction with different YIG interface-terminations. We find $G^{\uparrow \downarrow} \simeq 10^{14} \Omega^{-1} \mathrm{~m}^{-2}$ for both $\mathrm{Ag} \mid \mathrm{YIG}(111)$ and $\mathrm{Ag} \mid \mathrm{YIG}(001)$, with the real part dominating over the imaginary one, in stark contrast to the Stoner model (cf. Table 1). $G^{\uparrow \downarrow}$ depends only weakly on exposing different YIG(111) surface cuts to $\mathrm{Ag}$, which we attribute to the homogeneous distribution of magnetic atoms. The YFe termination of YIG(001) is a nearly compensated magnetic interface, but we still calculate a large spin mixing conductance. Finally, our results are two orders of magnitude larger than the experimental value found for Pt|YIG(111) [1]! The difference between $\mathrm{Ag}$ and Pt cannot account for this discrepancy: one would rather expect a larger $G^{\uparrow \downarrow}$ for Pt because of its higher conduction electron density.

The difference between the Stoner model and the first-principles calculations indicate that the spin-transfer torque physics at normal metal interfaces with YIG is very different from those with transition metals. Spin-transfer is equivalent to the absorption of a spin current at an interface that is polarized transversely to the magnetization direction. Magnetism in insulators is usually described in a local moment model. The physical picture of spin transfer appropriate for metals, viz. the destructive interference of precessing spins in the ferromagnet, then obviously fails. When the spin transfer acts locally on the magnetic ions, we expect no difference for the spin absorbed by a fully ordered interface with a large net magnetic moment or a

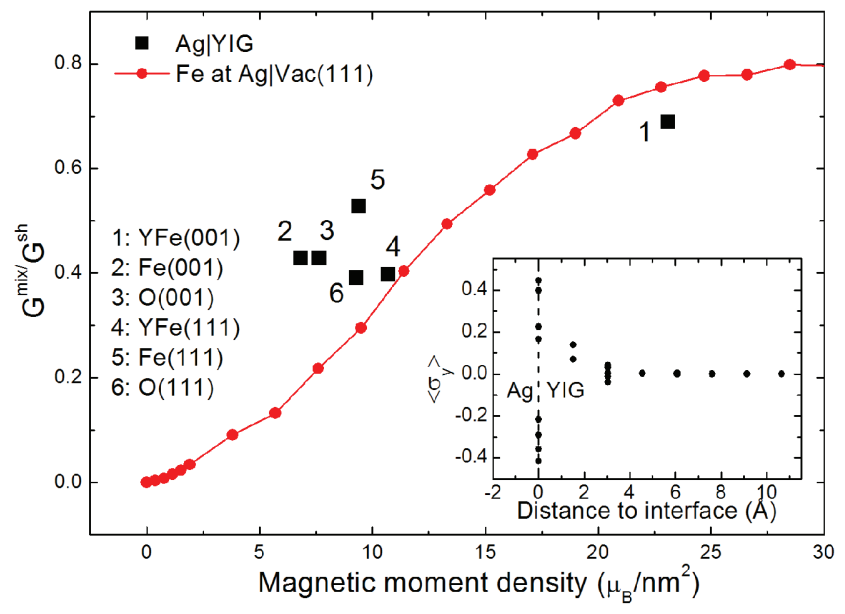

Fig. 4: $\operatorname{Re} G^{\uparrow \downarrow}$ as a function of interface magnetic moment density in $\mathrm{Ag} \mid \mathrm{YIG}$ compared with that of Fe atoms at the $\mathrm{Ag} \mid \mathrm{Vac}$ interface. Insert: Crystal-plane resolved spin density $\left\langle\sigma_{y}\right\rangle$ in arbitrary units for $\mathrm{Ag} \mid \mathrm{YIG}(001)$ with YFe-termination when fully polarized electrons are injected from the $\mathrm{Ag}$ side at mid-gap energy. The maximum magnetic moment density is $39 \mu_{B} / \mathrm{nm}^{2}$ as estimated from a full monolayer of $\mathrm{Fe}$ at the interface, in which the $\mathrm{Fe}$ atoms adopt the $\mathrm{Ag}$ structure with magnetic moment of $2.81 \mu_{B}$.

compensated one, in which the local moments point in opposite directions, as is indeed born out of our calculations.

In order to test the local moment paradigm, we consider non-conducting $\mathrm{Ag}|\operatorname{Vac}(4 \mathrm{~L})| \operatorname{Ag}(111)$ junctions. We now sprinkle one vacuum interface randomly with $\mathrm{Fe}$ atoms. At low densities the Fe atoms are weakly coupled and form local moments. The electronic structure is generated using the Coherent Potential Approximation (CPA) for interface disorder. A $10 \times 10$ lateral supercell with 100 atoms in one principle layer is used to model a magnetic impurity range from $1 \%$ to $80 \%$. The high density limit is a monolayer of Fe atoms in the fcc structure: $\mathrm{Ag}|\mathrm{Fe}(1 \mathrm{~L})| \operatorname{Vac}(3 \mathrm{~L}) \mid \mathrm{Ag}(111)$ with total magnetic moment of $2.81 \mu_{B}$ per $\mathrm{Fe}$ atom. So, the maximum magnetic moment density here is $39 \mu_{B} / \mathrm{nm}^{2}$. The results for the mixing conductances is summarized in Fig. 4. We find that the ratio of $G^{\uparrow \downarrow}$ to the $(\mathrm{Ag})$ Sharvin conductances monotonically increases with the Fe density at the $\mathrm{Ag} \mid \mathrm{Vac}$ interface. The increase is linear at small densities and saturates around $30 \mu_{B} / \mathrm{nm}^{2}$ due to interactions between neighboring moments. We find that $\operatorname{Re} G^{\uparrow \downarrow}$ of $\mathrm{Ag} \mid$ YIG and $\mathrm{Ag}|\mathrm{Fe}|$ vacuum agrees well for corresponding Fe densities at the interface, in strong support of the local moment model.

Since the mixing conductance is dominated by the local moments at the interface, we understand that the results are relatively stable against the difficulties density functional theory has for insulators. The variation of the band gap of the insulator as well as the band alignment with respect to normal metal changes the penetration of the 
spin accumulation, but since only the uppermost layers contribute this is of little consequence.

Table 4: $G^{\uparrow \downarrow}$ of a disordered Ag|YIG(001) interface with YFetermination. Directional disorder is introduced by flipping three majority Fe spins in the $2 \times 2$ super cell.

\begin{tabular}{lcc}
\hline \hline & $\operatorname{Re} G^{\uparrow \downarrow}\left(10^{14} \Omega^{-1} \mathrm{~m}^{-2}\right)$ & $\operatorname{Im} G^{\uparrow \downarrow}\left(10^{14} \Omega^{-1} \mathrm{~m}\right.$ \\
\hline clean & 3.010 & 0.302 \\
disorder & 3.145 & 0.382 \\
\hline \hline
\end{tabular}

Table 4 shows the effect of directional disorder of magnetic moments on the mixing conductance for Ag|YIG(001) with YFe-termination, for which the integrated surface magnetic moment density is close to zero. Here, we use a $2 \times 2$ lateral YIG supercell in which three magnetic moments are flipped to a negative value, amounting to a total surface magnetic moment of $-23.7 \mu_{B}$ per lateral unit cell. The directional disorder of magnetic moments at the interface slightly enhances $\operatorname{Re} G^{\uparrow \downarrow}$ (around 5\%), as indeed expected from the local moment picture.

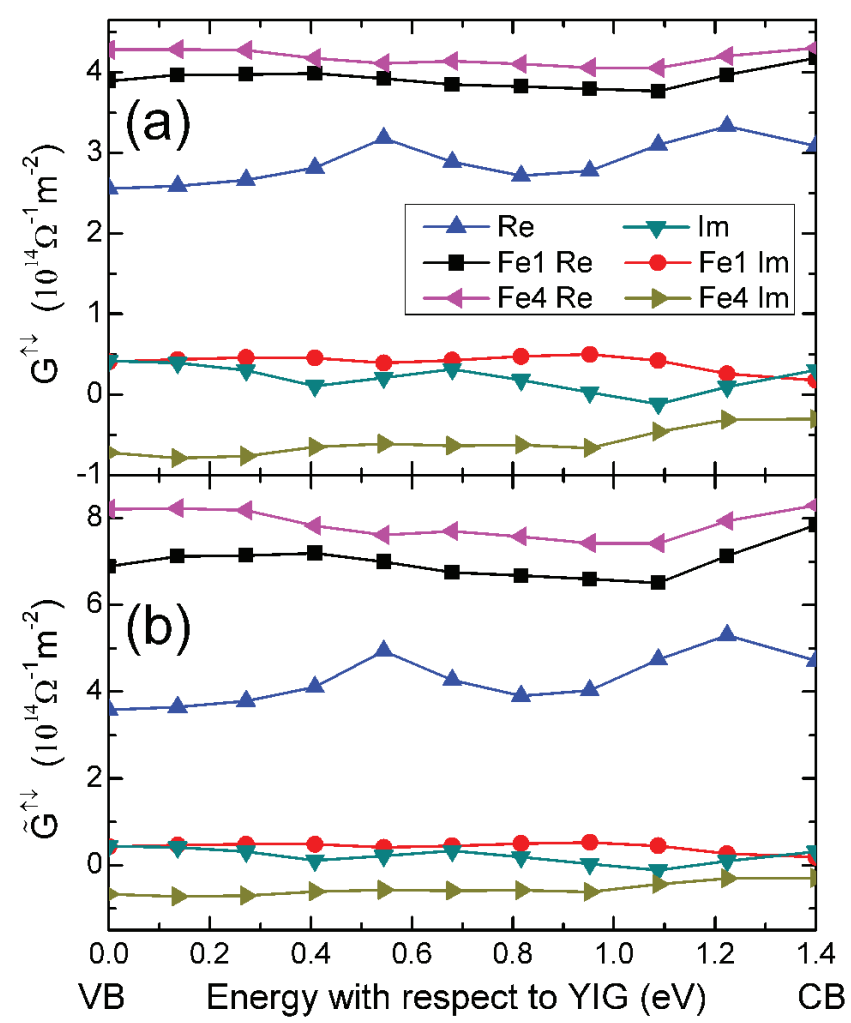

Fig. 5: Spin mixing conductance of $\mathrm{Ag}\left|\mathrm{Fe}_{n}\right| \mathrm{YIG}\left|\mathrm{Fe}_{n}\right| \mathrm{Ag}(001)$ with YFe termination for (a) ballistic and (b) diffusive transport (i.e. in the presence of Schep correction), where $n$ is the number for Fe monolayers inserted between $\mathrm{Ag}$ and YIG.

Inserting a thin ferromagnetic metallic layer between the normal metal and YIG should enhance the spin mixing conductance. In Fig. 5 (a), we show that inserting Fe atomic layers indeed increases $\operatorname{Re} G^{\uparrow \downarrow}$ by $40-65 \%$ up to the intermetallic $\mathrm{Ag} \mid \mathrm{Fe}$ value, which is close to the $\mathrm{Ag}$ Sharvin conductance.

In these calculation, the Ag reservoirs has been assumed to be ballistic. When the spin mixing conductance is small 2 relative to the Sharvin conductance, this is a valid approximation, but otherwise the diffusive nature of transport may not be be neglected. Since Re $G^{\uparrow \downarrow}$ turns out to be of the same order as $G_{A g}^{S h}$ we have to introduce the diffusive transport correction as introduced by Schep et al. as [28, 29]

$$
\frac{1}{\tilde{G}^{\uparrow \downarrow}}=\frac{1}{G^{\uparrow \downarrow}}-\frac{1}{2 G_{A g}^{S h}} .
$$

The results are shown in Fig. 5(b). We observe that the "Schep" correction enhances the spin mixing conductance by $20 \%$ for for the and about $90 \%$ for 4 nonolayers Fe insertions between $\mathrm{Ag}$ and YIG.

The spin transfer can be maximized by a high density of magnetic ions at the interfaces. In YIG we could not identify interface directions or cuts that are especially promising, but this could be different for other magnetic insulator, such as ferrites [8]. Slonczewski [8] uses a local moment model with a somewhat smaller exchange splitting $(0.5 \mathrm{eV})$ than found here; when defined as $\triangle(\vec{R})=\int_{\Omega_{W S}}\left(V_{x c}^{\downarrow}(\vec{R}, \vec{r})-V_{x c}^{\uparrow}(\vec{R}, \vec{r})\right) \rho(\vec{R}, \vec{r}) d \vec{r}$, where $\rho(\vec{R}, \vec{r})$ is the density of the evanescent wave function in YIG at mid-gap energy disregarding its spin splitting [30], $\Omega_{W S}$ the Wigner-Seitz sphere at the lattice site $\vec{R}$, and $V_{x c}^{\uparrow(\downarrow)}$ denotes the exchange-correlation potentials for spin-up (down) electronsthe exchange splitting felt by the Ag conduction electrons at the YIG interface is up to $\sim 3.0 \mathrm{eV}$. Since Slonzcewski focusses on the magnetization dynamics of the magnetic insulator we cannot carry out a quantitative comparison with his model here.

Conclusion. - In conclusion, we computed the spin mixing conductance $G^{\uparrow \downarrow}$ of the interface between silver and the insulating ferrimagnet Yttrium Iron Garnet (YIG). Re $G^{\uparrow \downarrow}$ is found to be of the order of $10^{14} \Omega^{-1} \mathrm{~m}^{-2}$, which is much larger than expected for a Stoner model, which indicates the importance of the local magnetic exchange field at the interface. On the other hand, $G^{\uparrow \downarrow}$ is not very sensitive to crystal orientation and interface cut. $\operatorname{Re} G^{\uparrow \downarrow}$ can be enhanced to around $40-65 \%$ of the fully metallic limit by inserting a monolayers of iron between $\mathrm{Ag}$ and YIG. The discrepancy between the measured and calculated mixing conductance might indicate previously unidentified interface contaminations that, when removed, would greatly improve the usefulness of magnetic insulators in spintronics.

$$
* * *
$$

We would like to thank Burkard Hillebrands, Eiji 
Saitoh, and Ken-ichi Uchida for stimulating discussions. This work was supported by National Basic Research Program of China (973 Program) under the grant No. 2011CB921803 and NSF-China grant No. 60825404, the EC Contract ICT-257159 "MACALO" and the Dutch FOM foundation. This research was supported in part by the Project of Knowledge Innovation Program (PKIP) of Chinese Academy of Sciences, Grant No. KJCX2.YW.W10.

Additional remark: After first submission of our manuscript arXiv:1103.3764, a manuscript was submitted and accepted by Physical Review Letters (Heinrich B. et al., Phys. Rev. Lett., 107 (2011) 066604) that reports a mixing conductance that is clearly enhanced compared to ref. [1], but still an order of magnitude smaller than our predictions.

\section{REFERENCES}

[1] Kajiwara Y., HariI K., Takahashi S., Ohe J., Uchida K., Mizuguchi M., Umezawa H., Kawai H., Ando K., Takanashi K., Maekawa S. and Saitoh E., Nature, 464 (2010) 262.

[2] Uchida K., Xiao J., Adachi H., Ohe J., Takahashi S., IEda J., Ota T., Kajiwara Y., Umezawa H., Kawai H., Bauer G. E. W., MaekawaS. and Saitoh E., Nat. Mater., 9 (2010) 894.

[3] Geller S. and Gilleo M. A., Acta Crystallogr., 10 (1957) 239.

[4] Cherepanov V., Kolokolov I. and Lvov V., Phys. Rep.-Rev. Sec. Phys. Lett., 229 (1993) 81.

[5] Serga A. A., Chumak A. V. and Hillebrands B., J. Phys. D: Appl. Phys., 43 (2010) 264002.

[6] Schneider T., Serga A. A., Leven B., Hillebrands B., Stamps R. L. and Kostylev M. P., Appl. Phys. Lett., 92 (2008) 022505.

[7] Saitoh E., Ueda M., Miyajima H. and Tatara G., Appl.Phys. Lett., 88 (2006) 182509.

[8] Slonczewski J. C., Phys. Rev. B, 82 (2010) 054403.

[9] Hatami M., Bauer G. E. W., Zhang Q. and Kelly P. J., Phys. Rev. Lett., 99 (2007) 066603.

[10] Yu H., Granville S., Yu D. P. and Ansermet J.-Ph., Phys. Rev. Lett., 104 (2010) 146601.

[11] Xiao J., Bauer G. E. W., Uchida K.-C., Saitoh E. and Maekawa S., Phys. Rev. B, 81 (2010) 214418.

[12] Adachi H., Ohe J., Takahashi S., Maekawa S., Phys. Rev. B, 83 (2011) 094410.

[13] Brataas A.,Bauer G. E. W. and Kelly P. J., Phys. Rep., 427 (2006) 157.

[14] Kimura T. and Otani Y., Phys. Rev. Lett., 99 (2007) 196604.

[15] Jedema F. J., Heersche H. B., Filip A. T., Baselmans J. J. A. and van Wees B. J., Nature, 416 (2002) 713.

[16] Andersen O. K., Jepsen O. and Glötzel D., Highlights of Condensed Matter Theory, edited by BASSANI F., Fumi F. and Tosi M. P. (North-Holland, Amsterdam) 1985 , p. 59

[17] Andersen O. K. and Jepsen O., Phys. Rev. Lett., 53 (1984) 2571
[18] Gunarson O., Jepsen O. and Andersen O. K., Phys. Rev. B, 27 (1983) 7144.

[19] Baettig P. and Oguchi T., Chem. Mater., 20 (2008) 7545 .

[20] Metselaar R. and Larsen P. K., Solid State Commun. 15 (1974) 291

[21] Wittekoek S., Popma T. J. A., Robertson J. M. and Bongers P. F., Phys. Rev. B, 12 (1975) 2777.

[22] Rodic D., Mitric M., Tellgren R., Rundlof H. and Kremonovic A., J. Magnet. Magnet. Mater., 191 (1999) 137.

[23] Pascard H., Phys. Rev. B, 30 (1984) 2299.

[24] Gilleo M. A., Ferromagnetic Materials, edited by Wohlfarth E.P., Vol. 2 (North-Holland, Amsterdam) 1980, p. 1

[25] Ching W. Y., Gu Z.-Q. and Xu Y.-N., J. Appl. Phys., 89 (2001) 6883.

[26] Rogalev A., Goulon J., Wilhelm F., Brouder Ch., Yaresko A., Youssef J. Ben and Indenbom M. V., J. Magn. Magn. Mater., 321 (2009) 3945.

[27] Fiorentini V. and Baldereschi A., Phys. Rev. B, 1995 (51) 17196.

[28] Schep K. M., van Hoof J. B. A. N., Kelly P. J., Bauer G. E. W., and Inglesfield J. E., Phys. Rev. B, 56 (1997) 10805.

[29] Bauer G. E. W., Schep K. M., Kelly P. J., and Xia K., J. Phys. D: Appl. Phys., 35 (2002) 2410.

[30] Gunnarsson O., J. Phys. F: Metal Phys., 6 (1976) 587. 